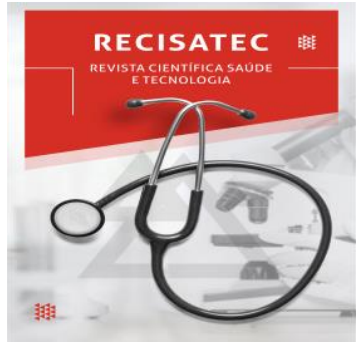

\title{
ACOMPANHAMENTO DE GESTANTES USUÁRIAS DE DROGA POR ENFERMEIROS DA ATENÇÃO BÁSICA
}

\section{FOLLOW-UP OF PREGNANT DRUG USERS BY PRIMARY CARE NURSES}

Elayne Alves dos Santos ${ }^{1}$, Ruana Dias Carvalho de Moraes², Raquel Vilanova Araújo³ $^{3}$

e23105

https://doi.org/10.53612/recisatec.v2i3.105

\section{RESUMO}

A gravidez é influenciada por diversos fatores, que podem alterar positiva ou negativamente a saúde da mãe e do feto. Destaca-se aqui o uso de drogas como sendo um fator negativo para a saúde do binômio mãe/feto. O estudo teve como objetivo descrever como é o acompanhamento de gestantes usuárias de droga por enfermeiros da atenção básica. Trata-se de um estudo descritivo de abordagem qualitativa realizada em 15 Unidades Básicas de Saúde (UBS) da zona Sul de TeresinaPI. Participaram da pesquisa 11 enfermeiros que prestaram assistência a gestantes usuárias de drogas e que aceitaram participar do estudo, para isso utilizou-se de um roteiro com perguntas semiestruturadas e de falas gravadas em celular, transcritas e analisadas com base na técnica de análise de conteúdo de Minayo. Emergiram duas categorias: 1- Ações do enfermeiro no acompanhamento de gestantes usuárias de drogas na atenção básica e 2- Dificuldades enfrentadas pelos enfermeiros no acompanhamento das gestantes usuárias de drogas. Das principais ações desenvolvidas pelos enfermeiros tem-se as consultas de enfermagem, solicitação de exames laboratoriais, agendamento de consultas, encaminhamento de risco, atividades educativas e de orientação à gestante. As principais dificuldades identificadas foram, a falta de continuidade do acompanhamento pré-natal pela gestante e elaboração de estratégias eficazes. Percebe-se que são muitas as dificuldades para o acompanhamento de gestantes usuárias de droga por enfermeiros. Destaca-se a importância da estruturação dos serviços de saúde e qualificação dos profissionais de saúde.

PALAVRAS-CHAVE: Gestante. Usuário de Drogas. Atenção Básica. Assistência Pré-Natal.

\section{ABSTRACT}

Pregnancy is influenced by several factors, which can positively or negatively alter the health of the mother and fetus. The use of drugs as a negative factor for the health of the mother/fetus binomial stands out here. The study aimed to describe how it is the follow-up of pregnant women who use drugs by primary care nurses. This is a descriptive study with a qualitative approach carried out in 15 Basic Health Units (UBS) in the South zone of Teresina-PI. The study included 11 nurses who provided care to pregnant women who were drug users and who agreed to participate in the study, for this purpose, using a script with semi-structured questions and the speeches recorded on cell phones, transcribed and analyzed based on Minayo's content analysis technique. Two categories emerged: 1Actions of nurses in the follow-up of pregnant women who use drugs in primary care and 2-Difficulties faced by nurses in the follow-up of pregnant women who use drugs. The main actions developed by nurses include nursing consultations, request for laboratory tests, scheduling appointments, risk referral, educational activities and guidance to pregnant women. The main difficulties identified were the lack of continuity of prenatal follow-up by the pregnant woman and the elaboration of effective strategies. It is noticed that there are many difficulties for the follow-up of pregnant women who use

\footnotetext{
1 Discente de Pós-graduação em Enfermagem Obstétrica do Instituto de Ensino Superior Múltiplo- IESM, TimonMA

${ }^{2}$ Discente de Pós-graduação em Enfermagem Obstétrica do Instituto de Ensino Superior Múltiplo- IESM, TimonMA

${ }^{3}$ Doutoranda em Enfermagem- Universidade Federal do Piauí- UFPI-PPG Enf. Mestre em Ciências em SaúdeCCSPI, Teresina-PI.
} 


\section{RECISATEC - REVISTA CIENTÍFICA SAÚDE E TECNOLOGIA ISSN 2763-8405}

ACOMPANHAMENTO DE GESTANTES USUÁRIAS DE DROGA POR ENFERMEIROS DA ATENÇÃO BÁSICA Elayne Alves dos Santos, Ruana Dias Carvalho de Moraes, Raquel Vilanova Araújo

drugs by nurses. The importance of structuring health services and the qualification of health professionals is highlighted.

KEYWORDS: Pregnant. Drug user. Basic Attention. Prenatal Assistance.

\section{CONSIDERAÇÕES INICIAIS}

No Brasil, o uso de drogas lícita e ilícita pela população feminina vem aumentando consideravelmente ao longo dos anos. Estima-se que aproximadamente $20 \%$ das mulheres façam uso de drogas durante a gravidez, configurado como um importante problema de saúde pública (MATA et al., 2019). A gravidez é influenciada por diversos fatores, que podem alterar positiva ou negativamente a saúde mãe e do feto. Quanto ao uso de drogas como um fator negativo, observouse o aumento de evidências científicas dos efeitos negativos relacionados ao consumo baixo a moderado durante a gestação, tendo em vista as complicações que pode causar como: hipoperfusão uteroplacentária, que pode diminuir o crescimento intrauterino, elevação dos índices de rotura prematura de membranas e descolamento prematuro de placenta. Pode ainda causar malformações no feto, abortos, aumento da mortalidade materna e fetal, e mortalidade neonatal (LIMA et al., 2015).

Promover a saúde destas gestantes é favorecer o ciclo gravídico-puerperal de modo que ele transcorra de maneira mais tranquila e segura. As consultas de pré-natal são de fundamental importância, bem como, as orientações na prevenção do uso de drogas na gestação e no puerpério, estas orientações devem continuar, a fim de conscientizar principalmente as gestantes dos perigos e consequências da droga durante a gravidez e no período puerperal, a assistência na atenção básica deve acompanhar a mulher desde o início da gravidez, para proporcionar, ao fim da gestação, o nascimento de um bebê saudável e a garantia do bem-estar materno (MAIA; PEREIRA; MENEZES, 2015).

Dessa forma, inúmeros fatores podem comprometer o processo natural da gestação. O uso de drogas surge, nesse contexto, como prática prejudicial que pode comprometer a saúde da mãe e do feto. Assim, a realização dessa pesquisa é marcada pela necessidade de se conhecer mais sobre o atendimento de gestantes usuárias de drogas na atenção básica, de modo a ampliar os conhecimentos a partir do que já foi publicado sobre os procedimentos realizados pelos enfermeiros nesses casos e buscar formas de melhoria do atendimento a esse público em particular.

O estudo permitirá que o tema seja mais amplamente discutido na comunidade científica, a partir de apresentações em eventos importantes na área da saúde e publicação do trabalho em periódicos de enfermagem, abrindo caminho para busca de estratégias que visem a melhoria do atendimento às gestantes usuárias de drogas na atenção básica. Apresenta ainda grande relevância acadêmica, pois os conhecimentos adquiridos por meio da pesquisa servirão para embasamento teórico-prático das ações realizadas a este público na atenção básica ao término da graduação. $O$ estudo tem como questão norteadora "quais as ações realizadas pelos enfermeiros no acompanhamento a gestantes usuárias de drogas na atenção básica?" Tendo como objetivos 


\section{RECISATEC - REVISTA CIENTÍFICA SAÚDE E TECNOLOGIA ISSN 2763-8405}

ACOMPANHAMENTO DE GESTANTES USUÁRIAS DE DROGA POR ENFERMEIROS DA ATENÇÃO BÁSICA Elayne Alves dos Santos, Ruana Dias Carvalho de Moraes, Raquel Vilanova Araújo

descrever as ações de acompanhamento do enfermeiro às gestantes usuárias de drogas na atenção básica e analisar as dificuldades que o enfermeiro encontra ao realizar o acompanhamento da gestante usuária de drogas na atenção básica.

\section{MÉTODO}

\subsection{Tipo de pesquisa}

Trata-se de um estudo descritivo de abordagem qualitativa realizado em uma (UBS) da zona Sul de Teresina-PI.

Foram incluídos na pesquisa 11 enfermeiros que trabalham na UBS e prestaram assistência a gestantes usuárias de drogas e que aceitaram participar do estudo mediante a assinatura do Termo de Consentimento Livre e Esclarecido (TCLE).

Para a coleta de dados utilizou-se um roteiro de perguntas semiestruturadas elaborada pelos pesquisadores, com dados de caracterização dos participantes e questões abertas relacionadas com o objeto de estudo. A entrevista ocorreu em local reservado para garantir sigilo e anonimato dos participantes, com data e hora marcada, de acordo com a disponibilidade do Enfermeiro e com duração aproximada de 15 minutos. As falas foram gravadas por meio de aparelho celular e os dados coletados no período de outubro a novembro de 2018.

Foram excluídos enfermeiros que estavam de férias, licença maternidade ou licença médica e que não possuem contrato efetivo com a Fundação Municipal de Saúde.

As falas foram transcritas e analisadas conforme a análise de conteúdo de Minayo (2013), seguindo as etapas: Pré-análise; exploração do material e tratamento dos resultados/inferência/interpretação.

A pesquisa foi autorizada pelo Comitê de Ética da Fundação Municipal de Saúde (CEP-FMS) e do Comitê de Ética e Pesquisa do Centro Universitário Santo Agostinho (CEP-UNIFSA), sob nº de parecer substanciado 2.979.772 e seguiu os princípios éticos da Resolução 466/12 do (CNS). Como garantia de sigilo e privacidade das informações fornecidas. Os enfermeiros foram identificados com nomes de flores, para garantir seu sigilo e anonimato.

\section{RESULTADOS E DISCUSSÃO}

\subsection{Caracterização dos participantes}

Dos 11 entrevistados, verificou-se que a maioria era do sexo feminino. 


\section{RECISATEC - REVISTA CIENTÍFICA SAÚDE E TECNOLOGIA ISSN 2763-8405}

ACOMPANHAMENTO DE GESTANTES USUÁRIAS DE DROGA POR ENFERMEIROS DA ATENÇ̃̃o BÁSICA Elayne Alves dos Santos, Ruana Dias Carvalho de Moraes, Raquel Vilanova Araújo

Quadro 1 - Caracterização dos participantes de acordo com o sexo, idade, e nível de formação. Teresina, 2018.

\begin{tabular}{|c|c|c|}
\hline Código & Sexo & Idade \\
\hline Azaleia & Feminino & 55 anos \\
\hline Rosa & Masculino & 38 anos \\
\hline Jasmim & Feminino & 35 anos \\
\hline Cravo & Feminino & 69 anos \\
\hline Copo de leite & Feminino & 48 anos \\
\hline Narciso & Feminino & 49 anos \\
\hline Girassol & Masculino & 27 anos \\
\hline Orquídea & Feminino & 30 anos \\
\hline Lírio & Masculino & 38 anos \\
\hline Tulipa & Feminino & 56 anos \\
\hline
\end{tabular}

Fonte: Dados pesquisa 2018.

\subsection{Categorização do Estudo}

A partir da análise parcial do conteúdo das falas dos entrevistados, emergiram as seguintes categorias: (1) A assistência de Enfermagem no acompanhamento de gestantes usuárias de drogas na atenção básica e (2) Dificuldades enfrentadas pelos Enfermeiros no acompanhamento da gestante usuária de drogas na atenção básica.

\subsection{A Assistência de Enfermagem no Acompanhamento de Gestantes Usuárias de Drogas na Atenção Básica}

Os profissionais evidenciaram em seus relatos a rotina de atendimento à gestante, com ênfase nas usuárias de drogas que procuram os serviços básicos de atenção à saúde, como observa-se nas falas:

[...] eu fiz várias visitas domiciliares, fiz a solicitações dos exames, orientei, pedi que elas viessem aqui a UBS, para agendar a ultrassom, encaminhei, fiz a avaliação de risco, pedi que ela fosse na maternidade também [...]. (Azaléia).

[...] a gente acompanha tentando manter a rotina do que é preconizado pelo programa do pré-natal, mantendo as consultas, consultas mensais até a $30^{\circ}$ semana, a partir daí encurtando essas [...]. (Rosa).

[...] a gente faz a consulta de rotina, faz a classificação de risco e dependendo da classificação de risco e dos atos clínicos [...]. (Cravo).

[...] A gente faz orientação sobre a droga e o risco para ela e para o bebê [...]. (Lírio).

Segundo Porto et al. (2015), uma considerável parcela das mulheres faz consumo de drogas durante a gravidez. Nesse sentido, observa-se que o consumo de drogas frequentemente tem aumentado nesse grupo, o que traz como consequências o aumento da evidência dos efeitos negativos relacionados ao consumo dessas substâncias durante a gestação. 


\section{RECISATEC - REVISTA CIENTÍFICA SAÚDE E TECNOLOGIA ISSN 2763-8405}

ACOMPANHAMENTO DE GESTANTES USUÁRIAS DE DROGA POR ENFERMEIROS DA ATENÇÃO BÁSICA Elayne Alves dos Santos, Ruana Dias Carvalho de Moraes, Raquel Vilanova Araújo

Nesse contexto, o acompanhamento pré-natal é essencial para controle da gestação dessas mulheres e prevenção de riscos materno-fetais. Lima et al. (2015) destaca a atuação do Enfermeiro no atendimento às gestantes usuárias de drogas, com realização de consulta pré-natal de baixo risco, solicitação de exames de rotina e prescrição de medicações de acordo com o protocolo do Ministério da Saúde ou do serviço, encaminhamento das gestantes de alto risco para o serviço de referência, realização de atividades educativas, registro dos dados da consulta e captação precoce para o pré-natal.

Observou-se como uma das ações descritas pelos entrevistados a realização de orientações a gestante usuária de drogas. Nesse sentido, Souza et al. (2014) ressalta a necessidade dessa ação nas unidades básicas de saúde, uma vez que na prática diária, muitas mulheres não recebem orientações adequadas quanto ao uso de substâncias que podem causar danos materno-fetais. Desta forma, o Enfermeiro deve apresentar-se como preparado para realizar ações educativas de orientação as gestantes, estimulando-as a realizarem todas as consultas de pré-natal para garantir sua saúde e do bebê.

Observou-se na fala de uma das participantes como é importante que o Enfermeiro busque estimular a construção e fortalecimento do vínculo entre a gestante e sua família, uma vez que essa é importante no processo de cuidado, como destacado no relato:

[...] A primeira coisa é estabelecer vínculos com a família, porque a pessoa com situação de vulnerabilidade que ela é usuária de drogas na maioria das vezes a gente não pode confiar ou contar com a palavra dela [...]. (Girassol).

Lima et al. (2017) destacam nesse contexto, que apesar do apoio que é oferecido pela equipe de saúde durante todo o período gestacional da mulher, não se deve descartar a importância que a família apresenta nesse momento, como facilitadora do processo de cuidado e garantia de melhor qualidade de vida para a mulher durante a gravidez.

\subsection{Dificuldades enfrentadas pelos Enfermeiros no acompanhamento da Gestante usuária de drogas na Atenção Básica}

O atendimento da gestante usuária de drogas na atenção básica foi visto ainda como um desafio para os Enfermeiros. Por meio dos relatos dos participantes observou-se que existem alguns fatores que dificultam esse acompanhamento. As participantes relataram como principal dificuldade para realização do acompanhamento às usuárias de drogas a ausência destas durante as consultas de acompanhamento, como é possível verificar nas falas a seguir:

[...] ela não cumpre as agendas, ela não faz as vacinas do pré-natal, ela não faz os suplementos vitamínicos de ferro, ácido fólico [...]. (Azaleia).

[...] as principais dificuldades que a gente como enfermeiros da estratégia enfrenta é exatamente o que a fuga dessa usuária dessa gestante. As vezes essas gestantes até iniciam o pré-natal, mas aí no meio do pré-natal elas começam a faltar as consultas. Você perde ela. [...]. (Rosa).

[...] A maior dificuldade é exatamente a vinda dessa gestante e continuidade no atendimento. [...] (Jasmim). 


\section{RECISATEC - REVISTA CIENTÍFICA SAÚDE E TECNOLOGIA ISSN 2763-8405}

ACOMPANHAMENTO DE GESTANTES USUÁRIAS DE DROGA POR ENFERMEIROS DA ATENÇÃO BÁSICA Elayne Alves dos Santos, Ruana Dias Carvalho de Moraes, Raquel Vilanova Araújo

[...] eu acho que é a própria iniciativa dela. As vezes elas vêm e abandonam o tratamento [...]. (Hortênsia).

[...]a nossa maior dificuldade atual é a de agente comunitário de saúde a gente tem só uma área coberta por agente comunitário de saúde fora as áreas que não tinham [...]. (Copo-de-leite).

A literatura aponta o uso de drogas como uma das principais causas de violência, problemas familiares e aumento das internações hospitalares na atualidade. Esse problema repercute diretamente na saúde das gestantes que estão sujeitas a sofrerem comprometimento do binômio mãe-filho como consequência do abuso dessas substâncias (MELO et al., 2014).

Autores como Porto et al. (2015) destacam a necessidade de intervenção profissional para estimular a realização do acompanhamento pré-natal de forma correta, uma vez que a vinculação precoce entre profissional e usuária é um fator determinante para a redução das vulnerabilidades na gestação relacionadas ao envolvimento com o álcool e outras drogas.

De acordo com Domingues et al. (2015), os problemas na adequação da assistência prénatal em âmbito nacional, sobretudo em relação a ausência das mulheres nas consultas de acompanhamento tem sido evidenciado em muitos estudos. Esse problema está associado a consequências negativas no período gestacional, como menor eficiência dos cuidados de saúde às gestantes e ocorrência de desfechos maternos e perinatais negativos, que contribuem para que a razão de mortalidade materna continue elevada e discrepante no país mesmo com os avanços ocorridos quanto ao desempenho dos sistemas de saúde.

Em concordância, Silva e Ferreira (2015) ressaltam que se deve estimular a realização do acompanhamento pré-natal por essas gestantes tendo em vista a importância que essa rotina, quando realizada de forma adequada, apresenta para a gestante e o feto, como a redução das taxas de morbimortalidade. A realização de exames nesse período contribui para a identificação de situações de risco e intervenções precoces.

Entre as dificuldades destacadas pelos Enfermeiros, verificou-se o fato de que ao sair do serviço de saúde muitas gestantes usuárias de drogas não tem casa para onde ir, ao passo que muitas vivem na rua ou não tem contato com os familiares, como verifica-se na fala:

[...] elas não têm casa, apesar de ter a casa da avó, a casa da mãe, mas elas não param em casa, só vivem na rua. Tem umas que moram na rua [...]. (Narciso).

[...] nem sempre a gente consegue que essa gestante retorne, principalmente quando ela está em situação de rua..., daí é mais difícil porque você não consegue encontrar ela em casa [....]. (Rosa).

[...] já tive caso também de pacientes em que não tem família e aí a gente tem que encontrar estratégias para tentar vincular essa gestante a unidade [...] (Girassol).

A despeito disso, Costa et al. (2015) destaca que a gravidez pode ser percebida como um fator de risco social no meio público, e como uma forma de fortalecimento da marginalidade e da pobreza a depender das situações do meio em que ocorre. Desta forma, a gestação quando ocorre em mulheres que moram na rua está relacionada a situação de vida precária e maior dificuldade de acesso aos serviços de saúde, o que é prejudicial para a saúde da mãe e bebê. 


\section{RECISATEC - REVISTA CIENTÍFICA SAÚDE E TECNOLOGIA ISSN 2763-8405}

ACOMPANHAMENTO DE GESTANTES USUÁRIAS DE DROGA POR ENFERMEIROS DA ATENÇÃO BÁSICA Elayne Alves dos Santos, Ruana Dias Carvalho de Moraes, Raquel Vilanova Araújo

Um dos grandes problemas para as gestantes usuárias de drogas, sobretudo aquelas que vivem em situação de rua, é o risco aumentado para as doenças sexualmente transmissíveis, principalmente pelos comportamentos promíscuos que podem apresentar sobretudo em relação a vida sexual. Assim, Ferreira, Lala e Mansour (2017) atribuem a fatores como elevação dos níveis hormonais e a imunossupressão, o risco de infecção aumentada para as doenças sexualmente transmissíveis durante a gravidez, devendo o enfermeiro orientar a gestante quando às práticas sexuais seguras e comportamentos de higiene.

A condição de moradora de rua favorece o aumento dos riscos para a gestante, que não conta com apoio e cuidados familiares para melhor qualidade de suas condições de vida. Santos (2017) destaca nesse cenário que as infecções sexualmente transmissíveis são consideradas um grave problema de saúde pública, com ocorrência estimada em mais de 340 milhões de novos casos no mundo por ano, que acomete homens e mulheres, principalmente aquelas em situação de rua.

Outra dificuldade observada pelos Enfermeiros foi em relação ao prejuízo à saúde causado pela própria droga, com aparecimento de comorbidades e o risco aumentado para a transmissão de doenças sexualmente transmissíveis, como é observado nos relatos:

[...] a questão da droga já debilita a própria paciente e faz com que ela tenha outras morbidades [...]. (Orquídea).

[...] O problema que a gente tem é que por ser usuária de droga geralmente tem os problemas associados, geralmente as usuárias de droga tem doença sexualmente transmissível devido as condições [...]. (Tulipa).

De acordo com Alves et al. (2017), a presença de doenças na gestação pode interferir significativamente na saúde materno-fetal, a exemplo de doenças crônicas como hipertensão e diabetes. Quando não tratadas corretamente podem levar a prognósticos pouco satisfatórios como a ocorrência de partos prematuros.

As complicações na saúde no período gestacional resultam em maiores riscos de complicações para a gestante e para o bebê. Entre as complicações que podem acometer a mulher grávida, Melo, Oliveira e Mathias (2015) destacam problemas como a sífilis congênita e hipertensão arterial gestacional, que se não diagnosticadas e tratadas precocemente podem desencadear consequências indesejáveis para a mãe e para o seu bebê. $O$ autor cita outros importantes problemas como malformações congênitas, infecções, pré-eclâmpsia e eclâmpsia, ganho de peso fetal inadequado e o nascimento prematuro.

Corroborando com os autores citados anteriormente, Moura et al. (2018) também destacam em seu estudo a estreita associação existente entre a presença de morbidades na gestante, como doenças hipertensivas, infecções e hemorragias, a desfechos negativos da gestação, onde destacase a mortalidade neonatal, fetal, o baixo peso ao nascer e a prematuridade.

$\mathrm{Na}$ gestação, o uso de drogas potencializa o risco de aparecimento de comorbidades. De acordo com Rodrigues et al. (2018), essas substâncias podem prejudicar a formação uterina e o desenvolvimento pós-natal. Drogas como o crack e a maconha ao serem consumidas espalham-se 


\section{RECISATEC - REVISTA CIENTÍFICA SAÚDE E TECNOLOGIA} ISSN 2763-8405

ACOMPANHAMENTO DE GESTANTES USUÁRIAS DE DROGA POR ENFERMEIROS DA ATENÇÃO BÁSICA Elayne Alves dos Santos, Ruana Dias Carvalho de Moraes, Raquel Vilanova Araújo

pelo organismo e apresentam efeitos cardiovasculares, neurológicos e pulmonares. Seu consumo está associado a morbidades como depressão e ansiedade, e problemas como violência, crimes e acidentes.

\section{CONSIDERAÇÕES FINAIS}

Observou-se que além das atividades básicas do pré-natal, os enfermeiros da UBS que assistem as gestantes usuárias de drogas, desenvolveram ações educativas e forneceram orientações quanto aos riscos do uso de drogas e possíveis complicações materno-fetal.

Dos maiores desafios para o acompanhamento das gestantes usuárias de drogas, é manter uma rotina de acompanhamento adequado no pré-natal já que a maioria abandona o acompanhamento, recusa-se a realizar exames e mantém-se distante dos serviços de saúde, além da insuficiência de agentes comunitários de saúde capacitados para trabalhar com essas gestantes. Destaca-se a importância de estratégias que permitam maior aproximação da gestante usuária de drogas com o serviço de atenção básica, de forma a garantir acompanhamento adequado por parte dos profissionais que prestam assistência a comunidade. Por perceber a escassez de pesquisas relacionadas ao problema, sugere-se a realização de estudos mais robustos relacionados ao tema em discussão.

\section{REFERÊNCIAS}

ALVES, N. C. C. et al. Complicações na gestação em mulheres com idade maior ou igual a 35 anos. Revista Gaúcha de Enfermagem, v. 38, n. 4, 2017. Disponível em: http://www.seer.ufrgs.br/RevistaGauchadeEnfermagem/article/download/78330/44951. Acesso em: 30 nov. 2018.

BARDIN, L. Análise de Conteúdo. Lisboa, Portugal: Edições 70, 2011.

BERTANI, A. L. et al. Prevenção do tabagismo na gravidez: importância do conhecimento materno sobre os malefícios para a saúde e opções de tratamento disponíveis. Jornal Brasileiro de Pneumologia, v. 41, n. 2, p. 175-181, 2015. Disponível em: http://www.jornaldepneumologia.com.br/trocaldioma.asp?varldioma=english\&varPagina=detalhe artig o.asp?id=2391. Acesso em: 19 abr. 2018.

COSTA, E. S. et al. Alterações fisiológicas na percepção de mulheres durante a gestação. Revista da Rede de Enfermagem do Nordeste, v. 11, n. 2, 2010 . Disponível em: http://www.redalyc.org/html/3240/324027970010/. Acesso em: 19 abr. 2018.

COSTA, S. L. et al. Gestantes em situação de rua no município de Santos, SP: reflexões e desafios para as políticas públicas. Saúde e Sociedade, v. 24, n. 3, p. 1089-102, 2015. Disponível em: https://doi.org/10.1590/S0104-12902015134769. Acesso em: 30 nov. 2018.

DOMINGUES, R. M. S. M. et al. Adequação da assistência pré-natal segundo as características maternas no Brasil. Revista Panamericana de Salud Pública, v. 37, p. 140-147, 2015. Disponível em: https://www.scielosp.org/scielo.php?pid=S1020-49892015000300003\&script=sci arttext\&tlng=en. Acesso em: 04 nov. 2018. 


\section{RECISATEC - REVISTA CIENTÍFICA SAÚDE E TECNOLOGIA ISSN 2763-8405}

ACOMPANHAMENTO DE GESTANTES USUÁRIAS DE DROGA POR ENFERMEIROS DA ATENÇÃO BÁSICA Elayne Alves dos Santos, Ruana Dias Carvalho de Moraes, Raquel Vilanova Araújo

FABRI, R. L.; SIQUEIRA, L. P.; FABRI, A. C. O. C. Aspectos gerais, farmacológicos e toxicológicos da cocaína e seus efeitos na gestação. Revista Eletrônica de Farmácia, v. 8, n. 2, p. 13, 2011. Disponível em: https://www.revistas.ufg.br/REF/article/viewFile/14960/9303. Acesso em: 08 abr. 2018.

FERREIRA, H.; LALA, E. R. P.; MANSOUR, F. R. Frequência de Papilomavirus humano (HPV) em gestantes. Biológicas \& Saúde, v. 7, n. 25, 2017. Disponível em: http://seer.perspectivasonline.com.br/index.php/biologicas e saude/article/download/1147/936.

Acesso em: 30 nov. 2018.

GIL, A. C. Como Elaborar Projetos de Pesquisa. 6. ed. São Paulo: Atlas, 2017. 192 p.

KASSADA D. S.; MARCON S. S.; WAIDMAN. M. A. P. Percepções e práticas de gestantes atendidas na atenção primária frente ao uso de drogas. Esc Anna Nery, v. 18, n. 3, p. 428-434, 2014. Disponível em: https://www.scielo.br/pdf/ean/v18n3/1414-8145ean-18-03-0428.pdf. Acesso em: 18 de ago. 2020.

KASSADA, D. S. et al. Prevalência do uso de drogas de abuso por gestantes. Acta Paulista de Enfermagem, v. 26, n. 5, 2013. Disponível em: http://www.redalyc.org/pdf/3070/307029420010.pdf. Acesso em: 15 mar. 2018.

LIMA, E. R. et al. Atuação e apoio de familiares no ciclo gravídico-puerperal: vivência prática em um grupo de gestantes. Revista Saúde-UNG-Ser, v. 11, n. 1 ESP, p. 54, 2017. Disponível em: http://revistas.ung.br/index.php/saude/article/download/3166/2277. Acesso em: 30 nov. 2018.

LIMA, L. P. M. et al. O papel do enfermeiro durante a consulta de pré-natal à gestante usuária de drogas. Espaço para Saúde, v. 16, n. 3, p. 39-46, 2015. Disponível em: http://espacoparasaude.fpp.edu.br/index.php/espacosaude/article/download/394/382. Acesso em: 05 nov. 2018.

MAIA, J. A.; PEREIRA, L. A.; MENEZES, F. A. Consequências do uso de drogas durante a gravidez. Revista Enfermagem Contemporânea, Salvador, v. 4, n. 2, p. 121-128, jul./dez, 2015. Disponível em: https://www5.bahiana.edu.br/index.php/enfermagem/article/view/664. Acesso em: 18 de ago. 2020.

MARANGONI, S. R; OLIVEIRA, M. L. F. Fatores desencadeantes do uso de drogas de abuso em mulheres. Texto \& Contexto Enfermagem, v. 22, n. 3, p. 662-70, 2013. Disponível em: http://www.scielo.br/pdf/tce/v22n3/v22n3a12. Acesso em: 06 abr. 2018.

MELO, E. C.; OLIVEIRA, R. R.; MATHIAS, T. A. F. Fatores associados à qualidade do pré-natal: uma abordagem ao nascimento prematuro. Revista da Escola de Enfermagem da USP, v. 49, n. 4, p. 540-549, 2015. Disponível em: http://www.redalyc.org/pdf/3610/361041522002.pdf. Acesso em: 04 dez. 2018.

MELO, V. H. et al. Uso de drogas ilícitas por gestantes infectadas pelo HIV. Rev Bras Ginecol Obstet., v. $36, \quad$ v. $12, \quad$ p. $555-61, \quad 2014 . \quad$ Disponível em: https://repositorio.observatoriodocuidado.org/bitstream/handle/369/1/rbgo.S010072032014001200555.pdf. Acesso em: 07 abr. 2018.

MINAYO, M. C. S. O desafio do conhecimento: pesquisa qualitativa em saúde. São Paulo: Hucitec, 2013.

MOTA, E. R. et al. Gestantes usuárias de substâncias psicoativas. Enferm Bras, v. 18, n. 4, p. 570-6, 2019. Disponível em: https://doi.org/10.33233/eb.v18i4.1649._Acesso em: 17 ago. 2020.

MOURA, B. L. A. et al. Internações por complicações obstétricas na gestação e desfechos maternos e perinatais, em uma coorte de gestantes no Sistema Único de Saúde no Município de São Paulo, 


\section{RECISATEC - REVISTA CIENTÍFICA SAÚDE E TECNOLOGIA ISSN 2763-8405}

ACOMPANHAMENTO DE GESTANTES USUÁRIAS DE DROGA POR ENFERMEIROS DA ATENÇÃO BÁSICA Elayne Alves dos Santos, Ruana Dias Carvalho de Moraes, Raquel Vilanova Araújo

Brasil. Cadernos de Saúde Pública, v. 34, p. e00188016, 2018. Disponível em: https://www.scielosp.org/scielo.php?pid=S0102-311X2018000105012\&script=sci arttext\&tlng=pt. Acesso em: 04 dez. 2018.

NAKAMURA, M. U.; KULAY JUNIOR, L; PASQUALE, M. Uso de fármacos na gravidez: benefício e custo. Revista Brasileira de Ginecologia e Obstetrícia, 2008. Disponível: http://www.scielo.br/pdf/rbgo/v30n1/a01v30n1. Acesso em: 08 abr. 2018.

NEVES, C.; MEDINA, J. L.; DELGADO, J. L. Alterações endócrinas e imuno-modulação na gravidez. Arquivos de medicina, v. 21, n. 5-6, p. 175-182, 2007. Disponível em: http://www.scielo.mec.pt/pdf/am/v21n5-6/v21n5-6a07.pdf. Acesso em: 19 abr. 2018.

PASTORE, A. P. W.; PRATES, C.; GUTIERREZ, L. L. P. Implicações da influenza A/H1N1 no período gestacional. Sci Med, v. 22, p. 53-8, 2012. Disponível em: http://www.maemecare.com.br/artigocientifico22.pdf. Acesso em: 09 abr. 2018.

PEIXOTO, C. R. et al. O pré-natal na atenção primária: o ponto de partida para reorganização da assistência obstétrica. Rev. enferm. UERJ, p. 286-291, 2011. Disponível em: http://www.facenf.ueri.br/v19n2/v19n2a19.pdf. Acesso em: 15 mar. 2018.

PENA, J. C. P. et al. Uso de álcool e tabaco na gestação: influência no peso do recém-nascido. Revista Saúde-UNG, v. 11, n. 1/2, p. 74-82, 2017. Disponível em: file:///C:/Users/Carla/Downloads/3070-9633-1-PB.pdf. Acesso em: 19 abr. 2018.

PORTELA, G. L. C. et al. Percepção da gestante sobre o consumo de drogas ilícitas na gestação. Rev. Eletrônica Saúde Mental Álcool, v. 9, n. 2, p. 58-63, may./aug. 2013. Disponível em. http://pepsic.bvsalud.org/pdf/smad/v9n2/pt 02.pdf. Acesso em: 18 de ago. 2020.

PORTO, P. N. et al. Acesso aos serviços de saúde: fatores associados ao envolvimento de gestantes com drogas. Revista Baiana de Enfermagem, v. 29, n. 4, p. 350-360, 2015. Disponível em: https://portalseer.ufba.br/index.php/enfermagem/article/viewFile/13832/pdf 16. Acesso em: 04 nov. 2018.

RAMOS, W. L. P. et al. Análise do uso de medicamentos durante a gestação em mães de pacientes portadores de malformações fetais. Saúde e Pesquisa, v. 1, n. 1, p. 59-64, 2008. Disponível em: http://periodicos.unicesumar.edu.br/index.php/saudpesq/article/download/709/581. Aceso em: 09 abr. 2018.

ROCHA, P. C. et al. Prevalência e fatores associados ao uso de drogas ilícitas em gestantes da coorte BRISA. Cad. Saúde Pública, Rio de Janeiro, v. 32, n. 1, p. e00192714, 2016. Disponível em: https://www.scielosp.org/article/ssm/content/raw/?resource ssm path=/media/assets/csp/v32n1/0102 -311X-csp-0102-311X00192714.pdf. Acesso em: 08 abr. 2018.

ROCHA, R. S. et al. Consumo de medicamentos, álcool e fumo na gestação e avaliação dos riscos teratogênicos. Revista Gaúcha de Enfermagem, v. 34, n. 2, p. 37-45, 2013. Disponível em: http://www.seer.ufrgs.br/RevistaGauchadeEnfermagem/article/viewFile/27191/26036. Acesso em: 05 abr. 2018.

RODRIGUES, A. P. et al. Como o crack e outras drogas podem influenciar no desenvolvimento de crianças que foram expostas durante o período gestacional. Revista Pesquisas e Práticas Psicossociais, v. 13, n. 1, p. 1-13, 2018. Disponível em: http://seer.ufsi.edu.br/index.php/revista ppp/article/viewFile/2856/1884. Acesso em: 04 dez. 2018.

SANTOS, C. S. et al. Avaliação de variáveis ao nascimento de recém-nascidos de mães usuárias de drogas. Saúde e meio ambiente: revista interdisciplinar, v. 5, n. 1, p. 4-13, 2016. Disponível em: http://www.periodicos.unc.br/index.php/sma/article/viewFile/862/643. Acesso em: 19 abr. 2018. 


\section{RECISATEC - REVISTA CIENTÍFICA SAÚDE E TECNOLOGIA ISSN 2763-8405}

ACOMPANHAMENTO DE GESTANTES USUÁRIAS DE DROGA POR ENFERMEIROS DA ATENÇÃO BÁSICA Elayne Alves dos Santos, Ruana Dias Carvalho de Moraes, Raquel Vilanova Araújo

SANTOS, J. N. et al. A orientação de enfermagem a gestantes que fazem uso de álcool e tabaco. Revista Recien-Revista Científica de Enfermagem, v. 4, n. 10, p. 5-11, 2014. Disponível em: http://recien.com.br/index.php/Recien/article/download/63/122. Acesso em: 30 nov. 2018.

SANTOS, M. V. S. Representações sociais de pessoas em situação de rua sobre cuidados para Infecções Sexualmente Transmissíveis. 2017. Dissertação (Mestrado) - Programa de PósGraduação em Enfermagem, da Escola de Enfermagem da Universidade Federal da Bahia, 2017. Disponível em: https://repositorio.ufba.br/ri/handle/ri/24382. Acesso em: 04 nov. 2018.

SILVA, C. P.; FERREIRA, F. V. G. Os desafios da gravidez na adolescência e a promoção da saúde. 2018. TCC (Graduação em Enfermagem) - Faculdade Integrada de Pernambuco - FACIPE, Recife, $2015 . \quad$ Disponível em: http://openrit.grupotiradentes.com/xmlui/bitstream/handle/set/2049/OS\%20DESAFIOS\%20DA\%20GR AVIDEZ\%20NA\%20ADOLESC\%C3\%8ANCIA\%20E\%20A\%20PROMO\%C3\%87\%C3\%830\%20DA\% 20SA\%C3\%9ADE.pdf?sequence=1. Acesso em: 04 nov. 2018.

SILVA, C. S.; TUFANIN, A. T. Alterações respiratórias e biomecânicas durante o terceiro trimestre de gestação: uma revisão de literatura. Revista Electônica Saúde E Ciência, v. 3, n. 2, 2013. Disponível em: http://www.rescceafi.com.br/vol3/n2/artigo\%2003\%20pags\%2028a37.pdf. Acesso em: 19 abr. 2018.

SOUZA, V. B.; ROECKER, S.; MARCON, S. S. Ações educativas durante a assistência pré-natal: percepção de gestantes atendidas na rede básica de Maringá-PR. Revista Eletrônica de Enfermagem, v. 13, n. 2, p. 199-210, 2011. Disponível em: https://www.fen.ufg.br/revista/v13/n2/v13n2a06.htm. Acesso em: 09 abr. 2018. 